\title{
THE PATHOLOGICAL EFFECTS OF CEREBRAL ARTERIOGRAPHY
} BY

\section{T. CRAWFORD}

\author{
From the Department of Pathology, St. George's Hospital Medical School, London
}

Cerebral arteriography is now carried out on a very large number of cases each year in the neurosurgical centres of the world. Usually this investigation is without harmful effect and the information gleaned commonly provides a precise diagnosis and leads to the institution of palliative or curative treatment. Nevertheless, there is inevitably some degree of damage to the arterial wall and in a very small proportion of cases harmful effects ensue. It seems important therefore that those who prescribe and carry out this procedure should be aware of the degree of damage commonly inflicted and of the occasional harmful effects which may follow. The method by which the arterial puncture wounds inflicted during arteriography heal " normally" has been described already (Crawford, 1956) and requires summarizing only briefly here. After some haemorrhage the track is filled by a fibrin plug. Endothelial cells proliferate and cover the central end of the plug by about the seventh day and thereafter the plug becomes organized by a dual mechanism from the lumen and from the adventitia similar to that seen in the organization of arterial

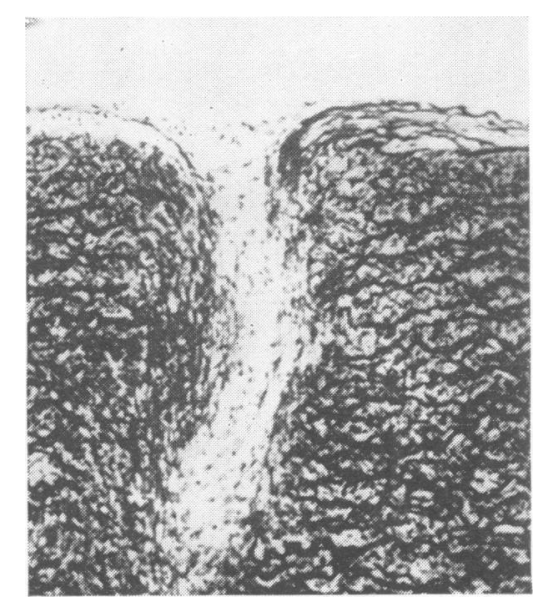

Fig. 1.- Site of arterial puncture made eight months before death. Elastic tissue has not regenerated. Weigert's elastic stain, $\times 65$. mural thrombi (Crawford and Levene, 1952). By about the 28th day organization is complete and a fibrous scar marks the site of puncture. Over the succeeding months a few elastic and smooth muscle fibres develop in the scar but even at eight months there is no proper reconstitution of the media or of the elastic laminae (Fig. 1).

This "normal" healing process occurs in the great majority of needle-tracks but departure from the simple healing process may occur in a variety of ways and produce effects of varying significance. It is with these "abnormal" effects of arterial puncture that the present paper is concerned. The descriptions which follow are based on necropsy material from 75 subjects on whom arteriography had been carried out at intervals varying from two hours to eight months before death. This material has come from many different sources and has not been confined to the necropsies from a single neurosurgical unit.

\section{Concealed Haemorrhage}

There is always some haemorrhage from the arterial puncture wound. In most cases it leads to no more than staining of the carotid sheath for a distance of three or four centimetres above and below the puncture; but quite often the entire carotid sheath is distended with blood from the arch of the aorta to the base of the skull (Fig. 2) and occasionally the blood breaks out from the sheaths and spreads widely amongst the structures of the neck and mediastinum (Fig. 3).

It has been noticed that haemorrhage is usually minimal in young subjects and most marked in elderly hypertensives or when the vessel has been transfixed by the needle (or punctured repeatedly) with the infliction of two or more wounds (Fig. 4).

These haemorrhages are such a striking feature at necropsies on recently punctured cases that it is surprising to the pathologist to learn that significant symptoms rarely occur. Transient pain, tenderness, and dysphagia may be admitted on questioning. Persistent damage has not been seen in this study. 


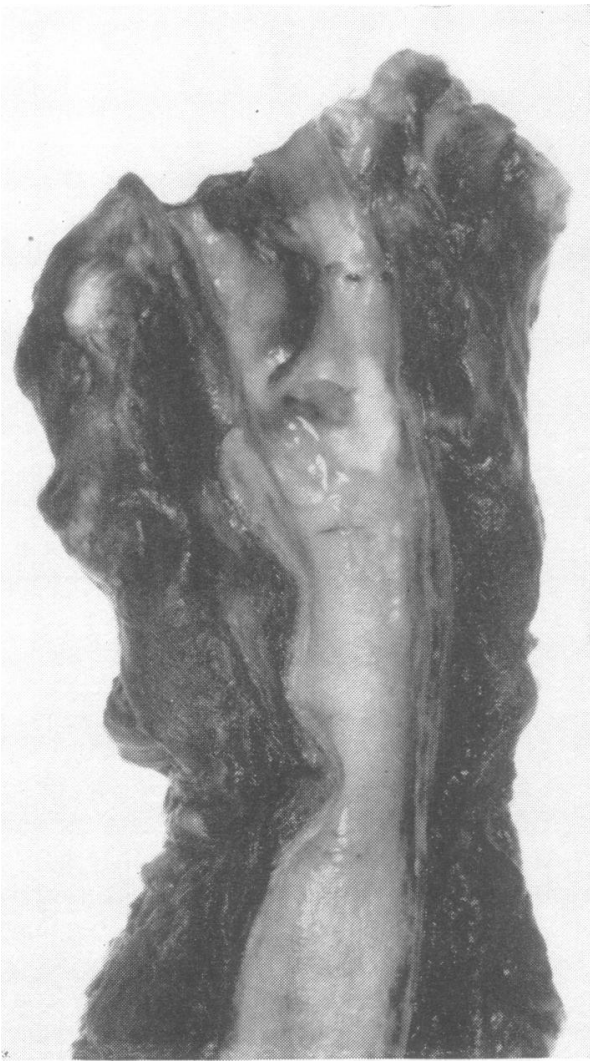

FIG. 2

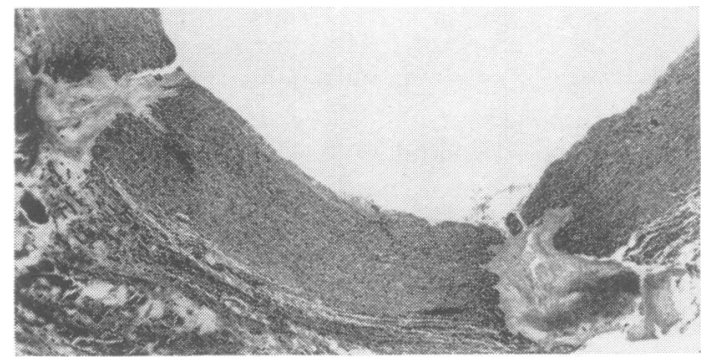

Fig. 4

Abscess Formation

Pyogenic infection has not been found in any of the necropsy material: but in several of the cases coming to necropsy within a week of arteriography the arterial wall was found to be heavily infiltrated with polymorphs in the neighbourhood of the needle track while a few showed sterile abscess formation in the adventitia and sheath of the artery (Fig. 5). There can be little doubt that these inflammatory reactions and sterile abscesses are due to injection of

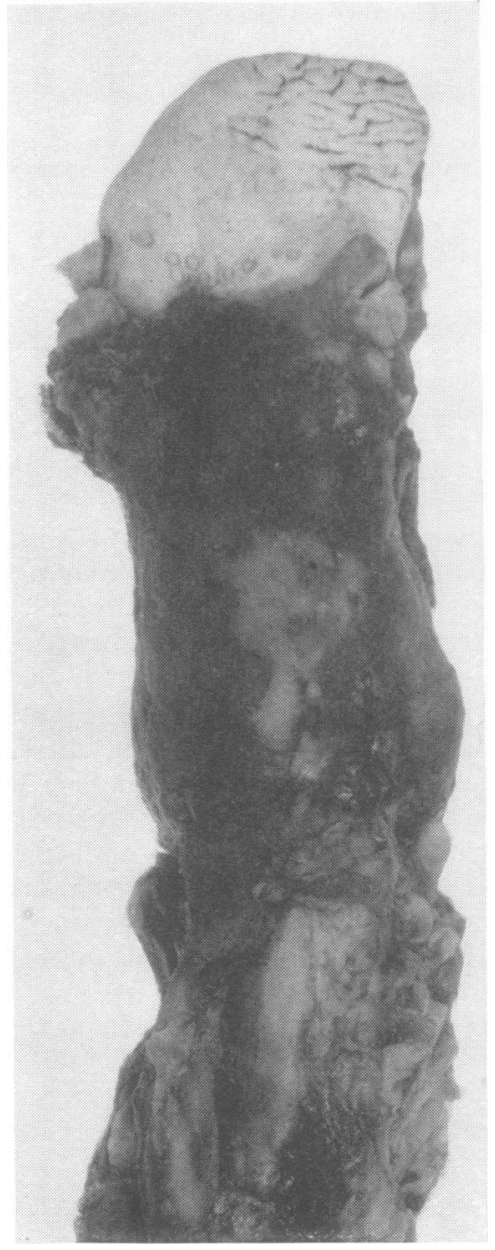

FIG. 3

FIG. 2.-Carotid sheath distended with blood. The arterial punct危e site can be seen just below the bifurcation.

FIG. 3.-Extensive haemorrhage into the tissues of the neck and mediastinum after carotid arteriography in a hypertensive womi.

Fig. 4.-Twin needle tracks in the wall of a carotid artery. Weigerfys elastic stain, $\times 25$.

contrast medium into the tissues of the arterial wall and sheath-an impression confirmed by the findiong of numbers of foamy macrophages amongst the polymorphs. Although harmful effects of thege reactions have not been recognized, damage to the important nerves in close anatomical relationsh to the carotid arteries could well result, and the further complication of thrombosis is more likePy when the vessel wall has been damaged by an intres mural injection. 


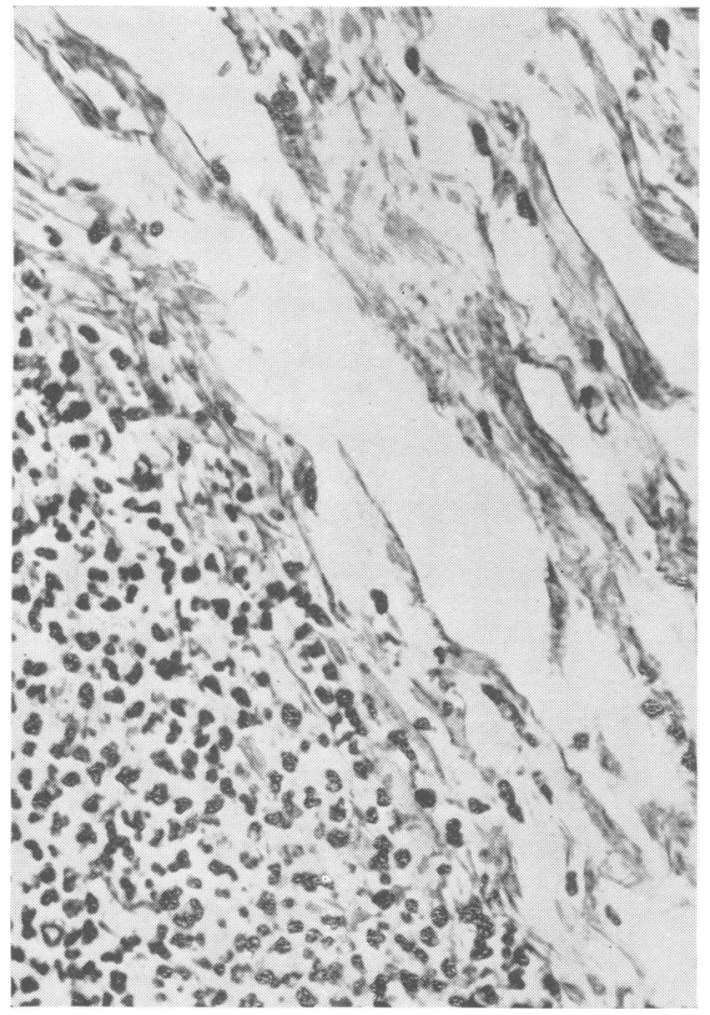

FIG. 5.-Sterile abscess in the carotid sheath. The arterial adventitia is seen above and to the left. Picro-Mallory, $\times 280$.

\section{Thrombosis}

This is perhaps the most important complication of arterial puncture for it is important not only in its direct effects but also as the precursor of embolism.

Occlusive Thrombosis.-Occlusive thrombosis affecting both common carotid arteries has been found once. In both vessels rapidly formed thrombus extended up from the puncture as far as the bifurcation. The patient was an elderly hypertensive man with massive intracranial haemorrhage and it was impossible to distinguish the effects of the thrombosis on the course of the disease. Obstruction of one carotid artery has been found four times. The subjects were all over 55 years of age and there was advanced atherosclerosis in the affected arteries. Here again the possible effects of the arterial obstruction were obscured by the progress of the severe subarachnoid haemorrhages for the localization of which the arteriographies had been performed but in one patient dying on the third day after arteriography early softening was found in the hemisphere on the affected side.
Mural Thrombosis.-Mural thrombosis at the site of arterial puncture is a frequent finding-in fact, some minor degree of it is an almost constant occurrence. Usually it consists merely of slight lateral extensions of the fibrin plug in the needle track over the adjacent parts of the intima. A thrombus of this type will soon be incorporated into the intima and will ultimately lead to nothing more serious than a localized thickening of that layer. Sometimes, however, the mural thrombus assumes an irregularly pedunculated or polypoid form (Fig. 6). The danger of embolism is then self evident.

\section{Cerebral Embolism}

Embolism results from detachment of a mural thrombus which has formed at the site of the puncture. The effects of embolism will, of course, vary with the size and site of lodgment of the embolus and clinically they are likely to be obscured by, or mistaken for, the progress of the disease process. In the present series of cases there was one instance of fatal embolism of the basilar artery following vertebral arteriography. In five further cases necropsy revealed multiple cerebral infarcts though death was due to the progress of the primary disease. In these cases the site of carotid arteriography was the only likely site of origin for emboli. The patients

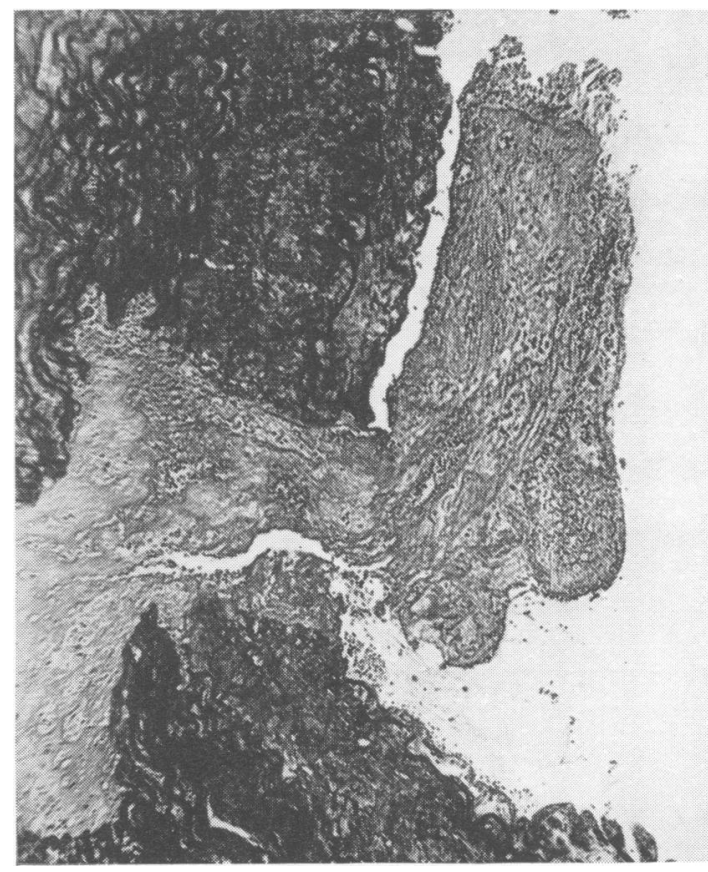

FIG. 6.-Mural thrombus with narrow attachment to the central end of the needle-track. Weigert's elastic stain, $\times 90$. 
were under investigation for subarachnoid haemorrhage. They showed rapid deterioration following arteriography, and necropsy confirmed that this was not due to recurrence of haemorrhage, though in each case the original damage from haemorrhage was of a grave nature.

\section{Aneurysm Formation}

Examination of the damaged vessel after recent arteriography punctures might lead one to anticipate that aneurysmal dilatation would be a frequent sequel but in fact trouble of this kind has not been encountered in the material studied. Two types of aneurysm-both of more pathological interest than clinical significance-have, however, been seen.

Traumatic False Aneurysm.-Two examples have been seen in which a small false aneurysm had developed through the needle track. One patient, a child aged 5 years, died 110 days after arteriography and at necropsy there was a berry-like nodule $1 \mathrm{~cm}$. in diameter on the wall of the common carotid artery at the site of puncture. Serial sectioning showed it to be a false aneurysm lying outside the media but communicating with the lumen by an endothelialized track (Fig. 7). The second example

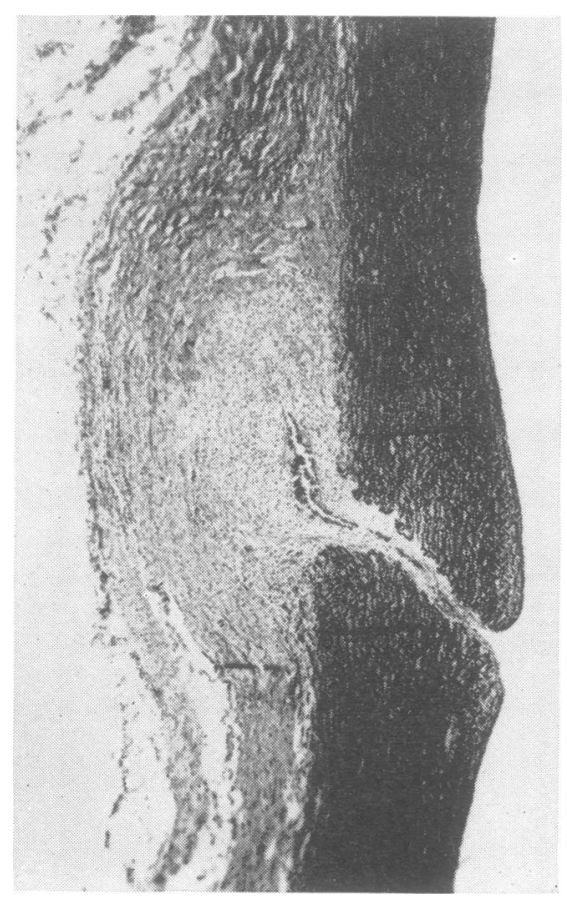

Fig. 7.-Traumatic false aneurysm at the site of needle-puncture 110 days before death. Weigert's elastic stain, $\times 25$. was a similar lesion in an elderly woman who died on the 28th day after arteriography. In neither cas did the aneurysm have any clinical effects.

Traumatic Dissecting Aneurysm.-CSmall dissecting aneurysms, consisting of blood-containing cavities in the substance of the media, have been seen to? follow arterial puncture on nine occasions. These lesions have varied from 0.5 to $4.0 \mathrm{~cm}$. in length. and have caused the formation of a rather prominen $\overline{\underline{E}}$ ridge on the intimal surface with sometimes some overlying mural thrombus. A typical example i $\$$ shown in Fig. 8. In two instances, in which deat?

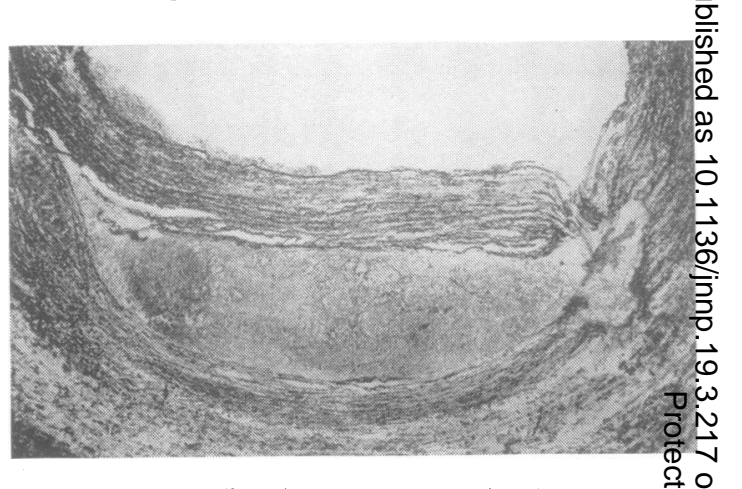

FIG. 8.-Traumatic dissecting aneurysm at the site of a neêdie puncture eight days before death. Weigert's elastic stain,

occurred on the 18 th and 24 th days respectivesy the dissecting spaces in the vessel wall had become extensively endothelialized. It is interesting to riteo that these traumatic lesions occur at a similar deptlp in the media to that at which spontaneous dissecting aneurysms develop.

\section{Discussion}

It is important to appreciate that the lesions which have been described in the preceding sections occurred amongst many thousands of patient subjected to arteriography. No quantitative assessment of the frequency of each type of lesion? can be made for many of the specimens were? submitted to the author precisely because of someunusual occurrence. Nevertheless, it is possible to recognize certain factors which increase the liability' to the development of complications. Advanced. age, hypertension, and severe atherosclerosis are three linked factors which come into this category and which have been present in a high proportiono of the cases in which thrombosis has occurred. On the technical side two further points emerge. In theo first place, multiplicity of punctures, as when the vessel is transfixed, or when repeated punctures ares made, greatly increases the amount of haemorrhage and the risk of thrombosis and embolism: and 
secondly, puncture close to the point of bifurcation of the common carotid artery carries a greater risk of complications than when the artery is entered a few centimetres lower down. This is associated with the fact that the region of the bifurcation is commonly the site of advanced and perhaps calcified atheromatous plaques. When the needle penetrates such a plaque an irregular gash in the wall may result and form a suitable nidus for platelet and fibrin deposition.

It would be presumptious for the pathologist to offer unsolicited advice to the clinician and radiologist in a matter of this kind, but if advice were sought it might perhaps take the following form: reserve arteriography for the study of grave disease; beware the elderly arteriosclerotic hypertensive subject; avoid puncturing the immediate neighbourhood of the carotid bifurcation; puncture the vessel once only and do not transfix it; consider the possibility of embolism when fresh neurological manifestations follow the investigation.

\section{Summary}

Lesions resulting from arterial puncture have been studied in necropsy material from 75 patients dying at intervals varying from a few hours up to eight months after cerebral arteriography. The most serious ill effects observed have been carotid thrombosis and cerebral embolism. It has not been possible to assess the frequency of these rare misfortunes. They are more liable to occur in elderly hypertensive arteriosclerotic subjects.

I wish to thank the many pathologists who have contributed necropsy material to this study, especially Drs. W. Blackwood, D. Dexter, N. F. C. Gowing, J. Guthrie, R. Pearce, and R. D. Teare. Thanks are also due to the clinicians who have placed their records at my disposal.

The work has been supported by a grant towards technical assistance from the Research Fund of St. George's Hospital.

\section{REFERENCES}

Crawford, T. (1956). J. Path. Bact. In the press. -, and Levene, C. I. (1952). Ibid., 64, 523. 